\title{
Biocompatibility and biodegradability of 2D materials: graphene and beyond
}

\author{
Cristina Martín, ${ }^{\mathrm{a}}$ Kostas Kostarelos, ${ }^{\mathrm{b}}$ Maurizio Prato ${ }^{\text {cde }}$ and Alberto Bianco ${ }^{* a}$
}

\author{
a University of Strasbourg, CNRS, Immunology, Immunopathology and Therapeutic \\ Chemistry, 67000 Strasbourg, France. E-mail: a.bianco@ibmc-cnrs.unistra.fr \\ ${ }^{b}$ Nanomedicine Lab, National Graphene Institute and Faculty of Biology, Medicine \& \\ Health, University of Manchester, Manchester, United Kingdom. \\ ${ }^{\mathrm{C} D}$ Department of Chemical and Pharmaceutical Sciences, University of Trieste, 34127 \\ Trieste, Italy \\ ${ }^{\mathrm{d} C a r b o n}$ Bionanotechnology Laboratory, CIC biomaGUNE, 20009 San Sebastian, Spain \\ ${ }^{\mathrm{e}}$ Basque Foundation for Science, Ikerbasque, 48013 Bilbao, Spain
}

\begin{abstract}
The potential risks associated with two-dimensional (2D) nanomaterials may cause serious concerns about their real applications and impact in biological systems. In addition, the demonstration of biodegradability of these flat nanomaterials is essential in living organisms. Here, we summarise the state-of-the-art in the field of biocompatibility and biodegradability of graphene-related materials (such as 2D materials like $\mathrm{MoS}_{2}, \mathrm{BN}$ or $\mathrm{WS}_{2}$ ). The impact of chemical functionalisation on the potential control of the biodegradability profile of these structures is also discussed.
\end{abstract}

\section{Introduction}

The development of two-dimensional (2D) materials, especially after the isolation of graphene in 2004, has stimulated enormous interest due to their unique properties. ${ }^{1}$ In fact, although graphene is probably the tip of the iceberg (since it is the most widely studied), there are other 2D materials (Fig. 1), which contribute to the mass of the same iceberg and are still unexploited and unexplored. ${ }^{2}$ The numerous applications of these layered nanomaterials including their functionalised derivatives are undeniable, due to their extraordinary physicochemical properties. ${ }^{3,4}$ Many reviews have previously summarised 2D materials spanning from their technological ${ }^{5-7}$ to their biomedical uses. ${ }^{8,9}$ Therefore, being aware of the environmental impact and possible risks on health of 2D nanomaterials it is imperative to address these concerns in a proactive manner, ${ }^{9,10}$ particularly in the case of bio-applications. In this context, this 
highlight focuses on the biocompatibility and biodegradability of 2D nanostructures reported over the last few years, including the role played by surface functionalisation.

\section{Biocompatibility}

The ability of materials to interact with cells, tissues or a living body without causing harmful effects is known as biocompatibility. Comparing the biocompatibility by cytotoxicity studies of 2Dnanomaterials, which differ in composition, size, number of layers, or functionalisation degree, helps to better understand the mechanisms responsible for any toxic effect, really assessing the hazard potential of these materials. Regarding graphene-based materials (GBMs), Pinto et al. reviewed in 2013 their biocompatibility based on existing work. ${ }^{11}$ The authors concluded that some studies reported the decrease or the slight decrease of bacterial and mammalian cell viability, after exposure to GBMs. However, systematic studies of the effect of the particle size on cell viability were still lacking. Moreover, knowledge of the long-term cytotoxicity of GBMs or the effect of these nanomaterials on cell signalling, among other biological processes, was just beginning to be unraveled. Soon after, it was found that pristine and functionalised graphene cause negligible $(>0.2 \%)$ hemolysis in red blood cells (up to $75 \mathrm{mg} \mathrm{mL}^{-1}$ ). ${ }^{12}$ In another interesting study, graphene synthesized through the chemical vapor deposition (CVD) method on copper foils was explored as a substrate to promote the cardiomyogenic differentiation process of mesenchymal stem cells. ${ }^{13}$ The nanomaterial did not exhibit any sign of cytotoxicity for the stem cell cultures, and the cell signalling molecules involved in the cardiomyogenic differentiation were upregulated. More recently, some of us showed that CVD graphene ${ }^{14}$ and graphene oxide papers ${ }^{15}$ could act as neuronal and other mammalian cell substrates favouring growth with no cytotoxic effects. Gurunathan et al. ${ }^{16}$ described the synthesis of graphene and GBMs, and highlighted their biocompatibility in the context of biological applications. The authors concluded that more studies on toxicity versus biocompatibility were needed, especially using in vivo models. Very recently we reported an extensive survey on safety assessment of GBMs by analysing the most up-to-date data on their biocompatibility. We classified a range of examples in different 3D graphs according to each particular GBM and each specific impact (i.e. on macrophages, on lungs, on the gastrointestinal tract). ${ }^{17}$ The general conclusion of this comprehensive work is that GBMs can be categorised according to their physicochemical characteristics and cannot be considered as a single type of materials. Indeed, GBMs differ according to three key parameters: the number of layers, dimension, and carbon-to-oxygen atomic ratio, and these parameters modulate the toxicity of each specific GBM, as postulated earlier by some of us. ${ }^{18,19}$ We observed a predictable pattern of effects for the selected examples, but there are still missing gaps to be filled with alternative GBMs for more systematic characterisation. 


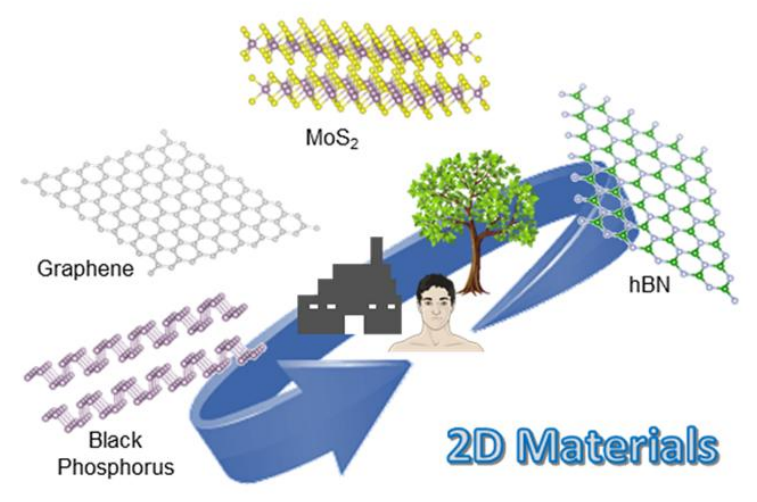

Fig. 1 Molecular schematics of some of the most developed 2D materials beyond graphene.

Regarding the influence of surface functionalisation on nanomaterial biocompatibility, many factors need to be taken into consideration. The GBM synthesis method in most cases tunesthefunctionalisation degreeofthesheets, influencingfor instance the cellular internalisation and other biological processes. It has been demonstrated that reduced graphene oxide ( $\mathrm{rGO}$ ) functionalised with a biocompatible biopolymer, which makes it highly stable in water, showed good cytocompatibility towards endothelial cells even at very high concentrations (i.e. $\left.100 \mathrm{mg} \mathrm{mL}{ }^{-1}\right){ }^{20}$ In another work, graphene nanoplatelets were modified with poly(vinyl alcohol) (PVA), hydroxyethyl cellulose (HEC), poly(ethylene glycol) (PEG), poly(vinyl pyrrolidone), chondroitin, glucosamine, and hyaluronic acid (Fig. 2). ${ }^{21}$ All materials resulted in low haemolysis up to $500 \mathrm{mg} \mathrm{mL}^{-}$ ${ }^{1}$, which further decreased after polymer adsorption, reaching the best results with PVA and HEC. However, differences regarding biocompatibility were observed, being improved using PVA. The authors explained the differences in terms of the encapsulation and agglomeration of the graphene platelets by PVA, decreasing the cell interaction/internalisation. In addition, the behaviour of few-layer graphene (FLG) was assessed during three months in vivo, ${ }^{22}$ finding that the PEGylation of FLG significantly reduced the histological abnormalities in comparison to the unmodified materials. Jasim et al. had previously shown that a very large proportion of intravenously injected thin GO sheets functionalised and radiolabelled with In-111 were able to be excreted rapidly in the urine without causing kidney (or other tissue) damage. ${ }^{23}$ In addition, the critical role played by the thickness of functionalised graphene oxide (GO) sheets in tissue accumulation and urinary excretion has been recently evaluated. ${ }^{24}$ This pilot study provides an initial correlation between GBM structures and pharmacological profiles to understand how 2D structures behave in vivo. Yang et al. previously reported a quantitative evaluation of the biodistribution of $\mathrm{GO}$ after administration using 125I-labeled nanosized GO further functionalised with PEG. ${ }^{25}$ The biodistribution revealed a higher accumulation in the spleen compared to that in the liver at all time points. In a slight contrast with these results, a clear improvement of biocompatibility both in vitro and in vivo has been demonstrated for GO after surface modification with poly(acrylic acid) or PEG. ${ }^{26}$ All these representative examples covering different types 
of GBMs illustrate how the characteristics of the starting material and its chemical modification influence their biological impact, eventually allowing the development of safer GBMs by-design. Similar to GBMs, the atomic composition, the exfoliation process and the lateral dimensions of transition metal dichalcogenides (TMDCs), hexagonal boron nitride (hBN, also termed "white graphene") or black phosphorus (BP) are key factors in determining their biocompatibility. ${ }^{27-30}$ The group of Pumera has studied the role played by the chalcogen atoms in the cytotoxicity of TDMCs. ${ }^{31-33}$ The differences in the chemical reactivity of each TMDC are related to the release of the chalcogens, resulting in higher toxicity. In general terms, selenium and vanadium play an important role in the toxicity, and ditellurides show higher cytotoxicity than disulphide containing materials. ${ }^{27}$ The exfoliation process is also important; however, its correlation with the levels of cytotoxicity is not very clear yet, since there are several works resulting in contrasting data. As it has been previously discussed for GBMs, the functionalisation of these alternative 2D nanomaterials can lead to control of their biocompatibility. ${ }^{34}$ Among the series of related papers, it has been reported that the functionalisation of $\mathrm{MoS}_{2}$ sheets with lipoic acid-modified PEG increased their physiological stability and biocompatibility. ${ }^{35}$ The concentration of the $2 \mathrm{D}$ nanomaterial or the type of cell lines used in the experiments also plays an important role. In fact, a few studies have shown dose-dependent toxicity using different cell lines. ${ }^{36}$ Dose-dependent toxicity has also been observed for BP sheets using human lung carcinoma epithelial cells, ${ }^{37}$ while BP quantum dots did not induce inflammatory responses. ${ }^{38}$ Concerning the in vivo impact of this type of materials, the most important studies today have focused on functionalised 2D materials. For example, iron oxide decorated $\mathrm{MoS}_{2}$ PEGylated nanosheets have been used for chelator-free radiolabelling and multimodal imaging guided photothermal therapy. ${ }^{39}$ This multifunctional conjugate was used for in vivo experiments, since the PEGylation endows the nanocomposite with enhanced biocompatibility and a more favourable pharmacokinetic profile. BP nanosheets have also been proven to be robust delivery platforms for cancer theranostics. ${ }^{40}$ The drug loaded PEGylated BP nanosheets showed excellent long circulation confirmed by pharmacokinetic experiments, good biocompatibility, and enhanced antitumor effects both in vitro and in vivo. In conclusion, the mechanisms and material parameters responsible for tissue damage induced by $2 \mathrm{D}$ materials still need to be thoroughly examined. More investigations need to be performed to reveal the possible health risks of both 2D nanomaterials and related composites and hydrids. ${ }^{41-43}$ 


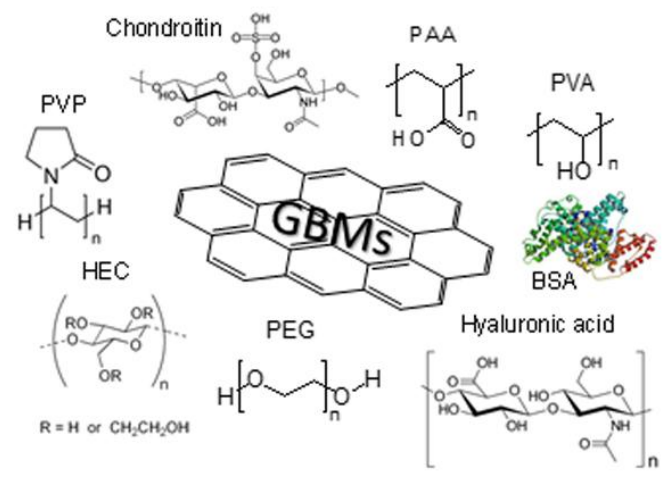

Fig. 2 Functionalisation strategies to enhance the biocompatibility of GBMs.

\section{Biodegradability}

Biodegradability refers to the ability of microorganisms to modify and alter the structure of a material by their metabolic or enzymatic action (Fig. 3). The complete clearance from the body and the biodegradation of 2D nanostructures need to be demonstrated in order for these materials to be approved for clinical use and to validate their safe use. Therefore, understanding the mechanisms leading to biodegradation has been associated to biocompatibility.

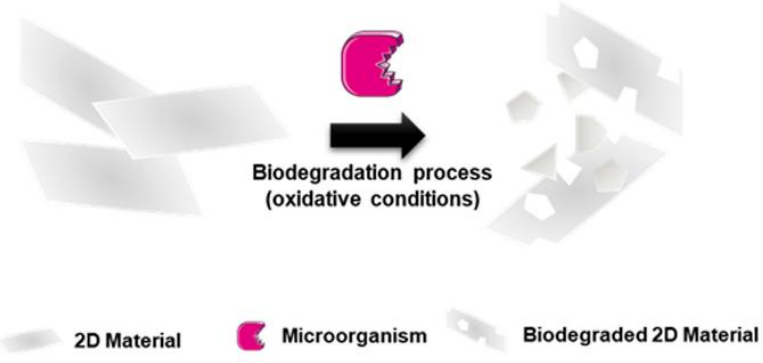

Fig. 3 Schematic representation of 2D material biodegradation.

Several techniques such as Raman, mass spectrometry and transmission electron microscopy can be used to assess biodegradation, particularly following the morphological and structural changes of the nanomaterials during this process. ${ }^{44}$ GBMs, and carbon nanomaterials in general, were assumed to be structurally persistent. However, subsequent work evidenced that oxidative enzymes (i.e. peroxidases) are able to catalyse the degradation of graphene oxide or carbon nanotubes in test tubes, in vitro and in vivo. ${ }^{45-48}$ In addition, our very recent review carefully evaluated the role of the material properties including the number of layers, the lateral dimension and the $\mathrm{C} / \mathrm{O}$ ratio in the degradation ability of each specific GBM (Fig. 4). ${ }^{17}$ In a comparative study it has been demonstrated that the degradation of GO sheets by hypochlorite was faster than that of 1D oxidised carbon nanotubes or nanohorns. ${ }^{49}$ The research performed in the last few years allowed us to confirm also 
the importance of the nanomaterial dispersibility, ${ }^{50}$ the synthetic strategy and the role played by surface functionalisation in the biodegradation process. Among GBMs, GO is the most studied 2D material in the biological context due to its versatile surface modification and the aqueous dispersibility. The investigation of the effect of surface coatings on the biodegradation of $\mathrm{GO}$ and its derivatives revealed that both PEG and BSA (bovine serum albumin) protect the material from degradation by horseradish peroxidase (HRP). ${ }^{51}$ In view of those results, the authors of the study designed an intermediate cleavable disulfide bond strategy that rendered the 2D nanomaterial biodegradable with negligible toxicity. Our group devised a new system to "attract" enzymes toward GO by functionalising the nanomaterial with coumarin and catechol, which are natural ligands of HRP. ${ }^{52}$ Not only GO but also single-layer graphene and few-layer graphene have been recently investigated. We have studied the biodegradability of these materials by human myeloperoxidase (MPO) and in the presence of degranulating human neutrophils, cells that are able to secrete high concentrations of MPO when activated. ${ }^{53}$ The degradation of both single- and fewlayer graphene was proved, concluding that these water-dispersed pristine carbon nanostructures are not biopersistent. Finally, we also demonstrated that alternative artificial enzymes, like DNAzymes consisting of a PS2.M-hemin complex that mimic HRP, can degrade GO. ${ }^{54}$ However, additional studies to test not only in vitro but also in vivo degradation and elimination of GBMs are necessary, in order to exclude their possible long-term accumulation and persistence. Interestingly, very little is still known on the biodegradation possibilities of other non-carbonaceous 2D materials like hBN, $\mathrm{MoS}_{2}$, graphitic $\mathrm{C}_{3} \mathrm{~N}_{4}$, 2D clay materials or BP monolayers. ${ }^{55-59}$ The possibility of HRP, MPO and photo-Fenton reaction to degrade hBN was assessed by our group in 2016.60 We saw that HRP does not degrade hBN up to 60 days, while partial oxidation was observed by using MPO after $35 \mathrm{~h}$ and nearly complete oxidation/degradation of hBN occurred by photoFenton reaction within $100 \mathrm{~h}$. We also examined in a different work the biodegradability of water dispersible pristine and functionalised $\mathrm{MoS}_{2}$ nanosheets. ${ }^{61}$ Interestingly, both nanostructures showed a much quicker degradation in the presence of low concentrations of $\mathrm{H}_{2} \mathrm{O}_{2}$ without any enzymes compared to HRP or MPO treatments. In fact, it has also been demonstrated that $\mathrm{MoS}_{2}$ nanosheets are thermodynamically and kinetically unstable in the presence of $\mathrm{O}_{2}$, being degraded under ambient conditions in various oxidising aqueous environments. ${ }^{62}$ The in vivo long-term biodistribution, excretion and toxicity of PEGylated TMDC nanosheets have also been reported. ${ }^{63}$ This study demonstrated that PEGylated TMDCs made of $\mathrm{MoS}_{2}$, $\mathrm{WS}_{2}$ and $\mathrm{TiS}_{2}$ can accumulate in the reticuloendothelial system after intravenous injection. However, only MoS $_{2}$-PEGylated TMDC was degraded and excreted within one month due to its different chemical properties compared to modified $\mathrm{WS}_{2}$ and $\mathrm{TiS}_{2}$. The degradation ability of these alternative 2D nanostructures renders feasible their further development in the creation of new hybrid materials able to combine biodegradability and biocompatibility with biomedical applications such as, for example, photothermal cancer therapy. ${ }^{64}$ 


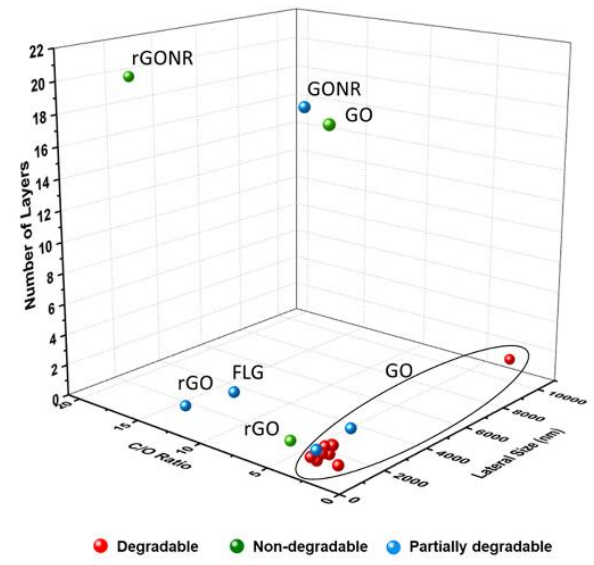

Fig. 4 Categorisation of the GBMs tested in studies on degradation reported in the literature (GONR, graphene oxide nanoribbons). Reprinted from ref. 17. Copyright 2018 American Chemical Society.

\section{Conclusion and future prospects}

Although a lot of research has been carried out in the field of biocompatibility and biodegradability of 2D nanomaterials, the progressive rise of such materials is indisputable, ${ }^{65}$ and the behaviour of the new $2 \mathrm{D}$ heterostructures that continue to appear needs to be assessed. Their safety evaluation should not only comprise the most recent GBMs that arise from novel strategies or that are characterised by modified surfaces, but also $2 \mathrm{D}$ materials like $\mathrm{Si} 2 \mathrm{BN},{ }^{66}$ borophene, ${ }^{67} \mathrm{ZnO},{ }^{68}$ among others that are of novel chemical consistency. In addition, the majority of the studies performed until now have been carried out in vitro, while critical validation tests should be extended to whole model organisms. Finally, the fundamental aspects and the mechanisms of their biological effects and actions are still poorly understood. The design of new artificial enzymes mimicking natural systems could help to fulfil this purpose. Therefore, more research has to be performed to cover the demand of rapid and high-content screening by generating large data sets that help to measure the safety of 2D materials. ${ }^{69}$ That is the only way to ensure the relevance of these materials in mass market technological and biomedical applications.

\section{Conflicts of interest}

There are no conflicts to declare.

\section{Acknowledgements}

The authors gratefully acknowledge the financial support from the EU GRAPHENE Flagship project (no. 696656 and no. 785219) and from the Agence Nationale de la Recherche (ANR) through the LabEx project Chemistry of Complex Systems (ANR-10- 
LABX-0026_CSC). This work was partly supported by the Centre National de la Recherche Scientifique (CNRS) and the International Center for Frontier Research in Chemistry (icFRC).

\section{Notes and references}

1 K. S. Novoselov, A. K. Geim, S. V Morozov, D. Jiang, Y. Zhang, S. V Dubonos, I. V Grigorieva and A. A. Firsov, Electric field effect in atomically thin carbon films., Science, 2004, 306, 666-9.

2 R. Mas-Ballesté, C. Gómez-Navarro, J. Gómez-Herrero and F. Zamora, 2D materials: to graphene and beyond, Nanoscale, 2011, 3, 20-30.

3 F. Yin, B. Gu, Y. Lin, N. Panwar, S. C. Tjin, J. Qu, S. P. Lau and K.-T. Yong, Functionalized 2D nanomaterials for gene delivery applications, Coord. Chem. Rev., 2017, 347, 77-97.

4 A. C. Ferrari, F. Bonaccorso, V. Fal'ko, K. S. Novoselov, S. Roche, P. Bøggild, S. Borini, F. H. L. Koppens, V. Palermo, N. Pugno, J. A. Garrido, R. Sordan, A. Bianco, L. Ballerini, M. Prato, E. Lidorikis, J. Kivioja, C. Marinelli, T. Ryhänen, A. Morpurgo, J. N. Coleman, V. Nicolosi, L. Colombo, A. Fert, M. Garcia-Hernandez, A. Bachtold, G. F. Schneider, F. Guinea, C. Dekker, M. Barbone, Z. Sun, C. Galiotis, A. N. Grigorenko, G. Konstantatos, A. Kis, M. Katsnelson, L. Vandersypen, A. Loiseau, V. Morandi, D. Neumaier, E. Treossi, V. Pellegrini, M. Polini, A. Tredicucci, G. M. Williams, B. Hee Hong, J.-H. Ahn, J. Min Kim, H. Zirath, B. J. van Wees, H. van der Zant, L. Occhipinti, A. Di Matteo, I. A. Kinloch, T. Seyller, E. Quesnel, X. Feng, K. Teo, N. Rupesinghe, P. Hakonen, S. R. T. Neil, Q. Tannock, T. Löfwander and J. Kinaret, Science and technology roadmap for graphene, related two-dimensional crystals, and hybrid systems, Nanoscale, $2015,7,4598-4810$.

5 J. Zhang and S. A. Meguid, Piezoelectricity of 2D nanomaterials: characterization, properties, and applications, Semicond. Sci. Technol., 2017, 32, 043006.

6 L. Peng, Z. Fang, Y. Zhu, C. Yan and G. Yu, Holey 2D Nanomaterials for Electrochemical Energy Storage, Adv. Energy Mater., 2018, 8, 1702179.

7 H. Wang, L. Yu, Y.-H. Lee, Y. Shi, A. Hsu, M. L. Chin, L.-J. Li, M. Dubey, J. Kong and T. Palacios, Integrated Circuits Based on Bilayer MoS 2 Transistors, Nano Lett., 2012, 12, 4674-4680.

8 R. Kurapati, K. Kostarelos, M. Prato and A. Bianco, Biomedical Uses for 2D Materials Beyond Graphene: Current Advances and Challenges Ahead, Adv. Mater., 2016, 28, 6052-6074.

9 T. P. Dasari Shareena, D. McShan, A. K. Dasmahapatra and P. B. Tchounwou, A Review on Graphene-Based Nanomaterials in Biomedical Applications and Risks in Environment and Health, Nano-Micro Lett., 2018, 10, 53.

10 M. Fojtů, W. Z. Teo and M. Pumera, Environmental impact and potential health risks of 2D nanomaterials, Environ. Sci. Nano, 2017, 4, 1617-1633.

11 A. M. Pinto, I. C. Gonçalves and F. D. Magalhães, Graphene-based materials biocompatibility: A review, Colloids Surfaces B Biointerfaces, 2013, 111, 188202.

12 A. Sasidharan, L. S. Panchakarla, A. R. Sadanandan, A. Ashokan, P. Chandran, C. M. Girish, D. Menon, S. V. Nair, C. N. R. Rao and M. Koyakutty, Hemocompatibility and Macrophage Response of Pristine and Functionalized Graphene, Small, 2012, 8, 1251-1263.

13 J. Park, S. Park, S. Ryu, S. H. Bhang, J. Kim, J.-K. Yoon, Y. H. Park, S.-P. Cho, S. Lee, B. H. Hong and B.-S. Kim, Graphene-Regulated Cardiomyogenic Differentiation Process of Mesenchymal Stem Cells by Enhancing the Expression of Extracellular Matrix Proteins and Cell Signaling Molecules, Adv. Healthc. Mater., 2014, 3, 176-181. 
14 N. P. Pampaloni, M. Lottner, M. Giugliano, A. Matruglio, F. D’Amico, M. Prato, J. A. Garrido, L. Ballerini and D. Scaini, Single-layer graphene modulates neuronal communication and augments membrane ion currents, Nat. Nanotechnol., 2018, 13, 755-764.

15 D. A. Jasim, N. Lozano, C. Bussy, I. Barbolina, A. F. Rodrigues, K. S. Novoselov and K. Kostarelos, Graphene-based papers as substrates for cell growth: Characterisation and impact on mammalian cells, FlatChem, 2018, 12, 17-25.

16 S. Gurunathan and J.-H. Kim, Synthesis, toxicity, biocompatibility, and biomedical applications of graphene and graphene-related materials, Int. J. Nanomedicine, 2016, 11, 1927.

17 B. Fadeel, C. Bussy, S. Merino, E. Vázquez, E. Flahaut, F. Mouchet, L. Evariste, L. Gauthier, A. J. Koivisto, U. Vogel, C. Martín, L. G. Delogu, T. Buerki-Thurnherr, P. Wick, D. Beloin-Saint-Pierre, R. Hischier, M. Pelin, F. Candotto Carniel, M. Tretiach, F. Cesca, F. Benfenati, D. Scaini, L. Ballerini, K. Kostarelos, M. Prato and A. Bianco, Safety Assessment of Graphene-Based Materials: Focus on Human Health and the Environment, ACS Nano, 2018, 12, 10582-10620.

18 K. Kostarelos and K. S. Novoselov, Exploring the Interface of Graphene and Biology, Science, 2014, 344, 261-263.

19 P. Wick, A. E. Louw-Gaume, M. Kucki, H. F. Krug, K. Kostarelos, B. Fadeel, K. A. Dawson, A. Salvati, E. Vázquez, L. Ballerini, M. Tretiach, F. Benfenati, E. Flahaut, L. Gauthier, M. Prato and A. Bianco, Classification Framework for GrapheneBased Materials, Angew. Chemie Int. Ed., 2014, 53, 7714-7718.

20 C. Cheng, S. Nie, S. Li, H. Peng, H. Yang, L. Ma, S. Sun and C. Zhao, Biopolymer functionalized reduced graphene oxide with enhanced biocompatibility via mussel inspired coatings/anchors, J. Mater. Chem. B, 2013, 1, 265-275.

21 A. M. Pinto, J. A. Moreira, F. D. Magalhães, I. C. Gonçalves and I. Gonçalves, Polymer surface adsorption as a strategy to improve the biocompatibility of graphene nanoplatelets., Colloids Surf. B. Biointerfaces, 2016, 146, 818-24.

22 A. Sasidharan, S. Swaroop, C. K. Koduri, C. M. Girish, P. Chandran, L. S. Panchakarla, V. H. Somasundaram, G. S. Gowd, S. Nair and M. Koyakutty, Comparative in vivo toxicity, organ biodistribution and immune response of pristine, carboxylated and PEGylated few-layer graphene sheets in Swiss albino mice: A three month study, Carbon, 2015, 95, 511-524.

23 D. A. Jasim, S. Murphy, L. Newman, A. Mironov, E. Prestat, J. McCaffrey, C. Ménard-Moyon, A. F. Rodrigues, A. Bianco, S. Haigh, R. Lennon and K. Kostarelos, The Effects of Extensive Glomerular Filtration of Thin Graphene Oxide Sheets on Kidney Physiology, ACS Nano, 2016, 10, 10753-10767.

24 D. A. Jasim, H. Boutin, M. Fairclough, C. Ménard-Moyon, C. Prenant, A. Bianco and K. Kostarelos, Thickness of functionalized graphene oxide sheets plays critical role in tissue accumulation and urinary excretion: A pilot PET/CT study, Appl. Mater. Today, 2016, 4, 24-30.

25 K. Yang, J. Wan, S. Zhang, Y. Zhang, S.-T. Lee and Z. Liu, In Vivo Pharmacokinetics, Long-Term Biodistribution, and Toxicology of PEGylated Graphene in Mice, ACS Nano, 2011, 5, 516-522.

26 M. Xu, J. Zhu, F. Wang, Y. Xiong, Y. Wu, Q. Wang, J. Weng, Z. Zhang, W. Chen and S. Liu, Improved In Vitro and In Vivo Biocompatibility of Graphene Oxide through Surface Modification: Poly(Acrylic Acid)-Functionalization is Superior to PEGylation, ACS Nano, 2016, 10, 3267-3281.

27 L. M. Guiney, X. Wang, T. Xia, A. E. Nel and M. C. Hersam, Assessing and Mitigating the Hazard Potential of Two-Dimensional Materials, ACS Nano, 2018, 12, 6360-6377.

28 Kenry and C. T. Lim, Biocompatibility and Nanotoxicity of Layered TwoDimensional Nanomaterials, ChemNanoMat, 2017, 3, 5-16.

29 S. Mateti, C. S. Wong, Z. Liu, W. Yang, Y. Li, L. H. Li and Y. Chen, Biocompatibility of boron nitride nanosheets, Nano Res., 2018, 11, 334-342.

30 M. Siepi, E. Morales-Narváez, N. Domingo, D. M. Monti, E. Notomista and A. Merkoçi, Production of biofunctionalized $\mathrm{MoS}_{2}$ flakes with rationally modified lysozyme: a biocompatible 2D hybrid material, 2D Mater., 2017, 4, 035007. 

Transition-Metal Dichalcogenides $\left(\mathrm{MoS}_{2}, \mathrm{WS}_{2}\right.$, and WSe ${ }_{2}$ ) is Lower Than That of Graphene and its Analogues, Chem. - A Eur. J., 2014, 20, 9627-9632.

32 N. M. Latiff, Z. Sofer, A. C. Fisher and M. Pumera, Cytotoxicity of Exfoliated Layered Vanadium Dichalcogenides, Chem. - A Eur. J., 2017, 23, 684-690.

33 H. L. Chia, N. M. Latiff, Z. Sofer and M. Pumera, Cytotoxicity of Group 5 Transition Metal Ditellurides ( $\mathrm{MTe}_{2} ; \mathrm{M}=\mathrm{V}, \mathrm{Nb}, \mathrm{Ta}$ ), Chem. - A Eur. J., 2018, 24, 206-211.

34 G. Qu, W. Liu, Y. Zhao, J. Gao, T. Xia, J. Shi, L. Hu, W. Zhou, J. Gao, H. Wang, Q. Luo, Q. Zhou, S. Liu, X.-F. Yu and G. Jiang, Improved Biocompatibility of Black Phosphorus Nanosheets by Chemical Modification, Angew. Chemie Int. Ed., 2017, 56, 14488-14493.

35 T. Liu, C. Wang, X. Gu, H. Gong, L. Cheng, X. Shi, L. Feng, B. Sun and Z. Liu, Drug Delivery with PEGylated $\mathrm{MoS}_{2}$ Nano-sheets for Combined Photothermal and Chemotherapy of Cancer, Adv. Mater., 2014, 26, 3433-3440.

36 S. Liu, Z. Shen, B. Wu, Y. Yu, H. Hou, X.-X. Zhang and H. Ren, Cytotoxicity and Efflux Pump Inhibition Induced by Molybdenum Disulfide and Boron Nitride Nanomaterials with Sheetlike Structure, Environ. Sci. Technol., 2017, 51, 1083410842.

37 N. M. Latiff, W. Z. Teo, Z. Sofer, A. C. Fisher and M. Pumera, The Cytotoxicity of Layered Black Phosphorus, Chem. - A Eur. J., 2015, 21, 13991-13995.

X. Mu, J.-Y. Wang, X. Bai, F. Xu, H. Liu, J. Yang, Y. Jing, L. Liu, X. Xue, H. Dai, Q. Liu, Y.-M. Sun, C. Liu and X.-D. Zhang, Black Phosphorus Quantum Dot Induced Oxidative Stress and Toxicity in Living Cells and Mice, ACS Appl. Mater. Interfaces, 2017, 9, 20399-20409.

39 T. Liu, S. Shi, C. Liang, S. Shen, L. Cheng, C. Wang, X. Song, S. Goel, T. E. Barnhart, W. Cai and Z. Liu, Iron Oxide Decorated MoS 2 Nanosheets with Double PEGylation for Chelator-Free Radiolabeling and Multimodal Imaging Guided Photothermal Therapy, ACS Nano, 2015, 9, 950-960.

40 W. Tao, X. Zhu, X. Yu, X. Zeng, Q. Xiao, X. Zhang, X. Ji, X. Wang, J. Shi, H. Zhang and L. Mei, Black Phosphorus Nanosheets as a Robust Delivery Platform for Cancer Theranostics, Adv. Mater., 2017, 29, 1603276.

41 D. McManus, S. Vranic, F. Withers, V. Sanchez-Romaguera, M. Macucci, H. Yang, R. Sorrentino, K. Parvez, S. Son, G. Iannaccone, K. Kostarelos, G. Fiori and C. Casiraghi, Water-based and Biocompatible 2D Crystal Inks: from Ink Formulation to All- Inkjet Printed Heterostructures, Nat. Nanotechnol., 2017, 12, 343.

42 C. Martín, S. Merino, J. M. González-Domínguez, R. Rauti, L. Ballerini, M. Prato and E. Vázquez, Graphene Improves the Biocompatibility of Polyacrylamide Hydrogels: 3D Polymeric Scaffolds for Neuronal Growth, Sci. Rep., 2017, 7, 10942.

43 R. F. Hossain, I. G. Deaguero, T. Boland and A. B. Kaul, Biocompatible, largeformat, inkjet printed heterostructure $\mathrm{MoS}_{2}$-graphene photodetectors on conformable substrates, npj 2D Mater. Appl., 2017, 1, 28.

44 M. Chen, X. Qin and G. Zeng, Biodegradation of Carbon Nanotubes, Graphene, and Their Derivatives, Trends Biotechnol., 2017, 35, 836-846.

45 J. Russier, L. Oudjedi, M. Piponnier, C. Bussy, M. Prato, K. Kostarelos, B. Lounis, A. Bianco and L. Cognet, Direct visualization of carbon nanotube degradation in primary cells by photothermal imaging., Nanoscale, 2017, 9, 4642-4645.

46 G. P. Kotchey, B. L. Allen, H. Vedala, N. Yanamala, A. A. Kapralov, Y. Y. Tyurina, J. Klein-Seetharaman, V. E. Kagan and A. Star, The Enzymatic Oxidation of Graphene Oxide, ACS Nano, 2011, 5, 2098-2108.

47 G. P. Kotchey, S. A. Hasan, A. A. Kapralov, S. H. Ha, K. Kim, A. A. Shvedova, V. E. Kagan and A. Star, A Natural Vanishing Act: The Enzyme-Catalyzed Degradation of Carbon Nanomaterials, Acc. Chem. Res., 2012, 45, 1770-1781.

48 K. Bhattacharya, S. P. Mukherjee, A. Gallud, S. C. Burkert, S. Bistarelli, S. Bellucci, M. Bottini, A. Star and B. Fadeel, Biological interactions of carbon-based nanomaterials: From coronation to degradation., Nanomedicine, 2016, 12, 33351. 
L. Newman, N. Lozano, M. Zhang, S. lijima, M. Yudasaka, C. Bussy and K. Kostarelos, Hypochlorite degrades 2D graphene oxide sheets faster than 1D oxidised carbon nanotubes and nanohorns, npj 2D Mater. Appl., 2017, 1, 39. R. Kurapati, J. Russier, M. A. Squillaci, E. Treossi, C. Ménard-Moyon, A. E. Del RioCastillo, E. Vazquez, P. Samorì, V. Palermo and A. Bianco, DispersibilityDependent Biodegradation of Graphene Oxide by Myeloperoxidase, Small, 2015, 11, 3985-3994.

51 Y. Li, L. Feng, X. Shi, X. Wang, Y. Yang, K. Yang, T. Liu, G. Yang and Z. Liu, Surface Coating-Dependent Cytotoxicity and Degradation of Graphene Derivatives: Towards the Design of Non-Toxic, Degradable Nano-Graphene, Small, 2014, 10, 1544-1554.

52 R. Kurapati, F. Bonachera, J. Russier, A. R. Sureshbabu, C. Ménard-Moyon, K. Kostarelos and A. Bianco, Covalent chemical functionalization enhances the biodegradation of graphene oxide, 2D Mater., 2017, 5, 015020.

53 R. Kurapati, S. P. Mukherjee, C. Martín, G. Bepete, E. Vázquez, A. Pénicaud, B. Fadeel and A. Bianco, Degradation of Single-Layer and Few-Layer Graphene by Neutrophil Myeloperoxidase, Angew. Chemie Int. Ed., 2018, 57, 11722-11727.

54 R. Kurapati and A. Bianco, Peroxidase mimicking DNAzymes degrade graphene oxide, Nanoscale, 2018, 10, 19316-19321.

55 H. Wang, X. Yang, W. Shao, S. Chen, J. Xie, X. Zhang, J. Wang and Y. Xie, Ultrathin Black Phosphorus Nanosheets for Efficient Singlet Oxygen Generation, J. Am. Chem. Soc., 2015, 137, 11376-11382.

56 S. Presolski and M. Pumera, Covalent functionalization of $\mathrm{MoS}_{2}$, Mater. Today, 2016, 19, 140-145.

57 S. Wang, Y. Wu, R. Guo, Y. Huang, S. Wen, M. Shen, J. Wang and X. Shi, Laponite Nanodisks as an Efficient Platform for Doxorubicin Delivery to Cancer Cells, Langmuir, 2013, 29, 5030-5036.

58 M. H. Khan, Z. Huang, F. Xiao, G. Casillas, Z. Chen, P. J. Molino and H. K. Liu, Synthesis of Large and Few Atomic Layers of Hexagonal Boron Nitride on Melted Copper, Sci. Rep., 2015, 5, 7743.

59 M. Xu, T. Liang, M. Shi and H. Chen, Graphene-Like Two-Dimensional Materials, Chem. Rev., 2013, 113, 3766-3798.

60 R. Kurapati, C. Backes, C. Ménard-Moyon, J. N. Coleman and A. Bianco, White Graphene undergoes Peroxidase Degradation, Angew. Chemie, 2016, 128, 55965601.

61 R. Kurapati, L. Muzi, A. P. R. de Garibay, J. Russier, D. Voiry, I. A. Vacchi, M. Chhowalla and A. Bianco, Enzymatic Biodegradability of Pristine and Functionalized Transition Metal Dichalcogenide MoS 2 Nanosheets, Adv. Funct. Mater., 2017, 27, 1605176.

62 Z. Wang, A. von dem Bussche, Y. Qiu, T. M. Valentin, K. Gion, A. B. Kane and R. $\mathrm{H}$. Hurt, Chemical Dissolution Pathways of $\mathrm{MoS}_{2}$ Nanosheets in Biological and Environmental Media., Environ. Sci. Technol., 2016, 50, 7208-17.

63 J. Hao, G. Song, T. Liu, X. Yi, K. Yang, L. Cheng and Z. Liu, In Vivo Long-Term Biodistribution, Excretion, and Toxicology of PEGylated Transition-Metal Dichalcogenides $\mathrm{MS}_{2}$ (M = Mo, W, Ti) Nanosheets, Adv. Sci., 2017, 4, 1600160.

64 J. Shao, H. Xie, H. Huang, Z. Li, Z. Sun, Y. Xu, Q. Xiao, X.-F. Yu, Y. Zhao, H. Zhang, $\mathrm{H}$. Wang and P. K. Chu, Biodegradable black phosphorus-based nanospheres for in vivo photothermal cancer therapy, Nat. Commun., 2016, 7, 12967.

65 N. Mounet, M. Gibertini, P. Schwaller, D. Campi, A. Merkys, A. Marrazzo, T. Sohier, I. E. Castelli, A. Cepellotti, G. Pizzi and N. Marzari, Two-dimensional materials from high-throughput computational exfoliation of experimentally known compounds, Nat. Nanotechnol., 2018, 13, 246-252.

66 A. N. Andriotis, E. Richter and M. Menon, Prediction of a new graphenelike Si 2 BN solid, Phys. Rev. B, 2016, 93, 081413.

67 V. Wang and W. T. Geng, Lattice Defects and the Mechanical Anisotropy of Borophene, J. Phys. Chem. C, 2017, 121, 10224-10232.

68 H. Ta, L. Zhao, D. Pohl, J. Pang, B. Trzebicka, B. Rellinghaus, D. Pribat, T. Gemming, Z. Liu, A. Bachmatiuk, M. Rümmeli, H. Q. Ta, L. Zhao, D. Pohl, J. Pang, 
B. Trzebicka, B. Rellinghaus, D. Pribat, T. Gemming, Z. Liu, A. Bachmatiuk and M. H. Rümmeli, Graphene-Like ZnO: A Mini Review, Crystals, 2016, 6, 100.

69 Y. Li, J. Wang, F. Zhao, B. Bai, G. Nie, A. E. Nel and Y. Zhao, Nanomaterial libraries and model organisms for rapid high-content analysis of nanosafety, Natl. Sci. Rev., 2018, 5, 365-388. 


\section{Biographies}

\section{Cristina Martín}

Cristina Martín finished her Chemistry studies in 2012 in the University of Castilla-La Mancha (Spain) and obtained her PhD in Chemistry in 2016 from the Universities of Trieste (Italy) and Castilla-La Mancha, working under the co-supervision of Prof. Maurizio Prato and Prof. Ester Va 'zquez. During her PhD, she spent 3 months at the University of Brighton (UK) under the supervision of Prof. Matteo Santin. As a postdoctoral researcher she worked in 2016-2017 at IRICA (Spain). In October 2017 she joined the group of Dr Alberto Bianco as postdoc. Her research focus is mainly on carbon nanostructures and their biodegradation and biomedical applications.

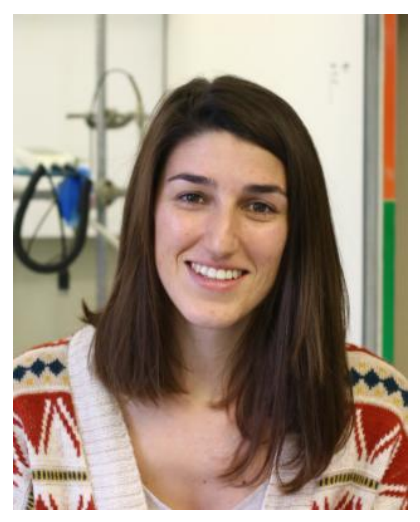

\section{Kostas Kostarelos}

Kostas is Chair of Nanomedicine at the University of Manchester and Visiting Professor at University College London (UCL). He obtained his PhD from the Department of Chemical Engineering at Imperial College London and carried out his postdoctoral training in various medical institutions (UCSF, Memorial Sloan-Kettering, Cornell University) in the USA. He has been faculty at Weill Cornell Medical College; Imperial College London and UCL. He has been an invited Fellow of the Royal Society of Chemistry (FRSC), the Royal Society of Medicine (FRSM), and the Royal Society of Arts (FRSA).

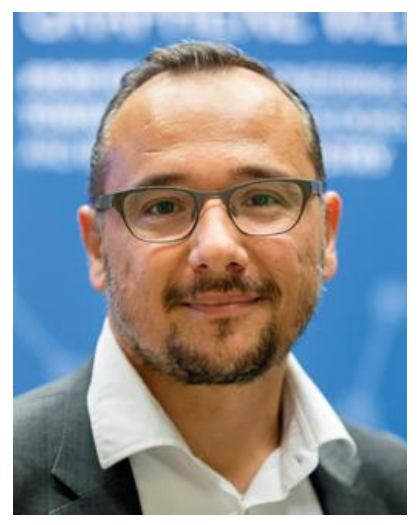




\section{Maurizio Prato}

Maurizio Prato graduated in Padova, where he was appointed Assistant Professor in 1983. He moved to Trieste in 1992, to become Professor in 2000. In 2015 he joined CICbiomaGUNE, Spain, as Ikerbasque Research Professor. He worked at Yale and University of California, Santa Barbara. He was the recipient of an ERC Advanced Research Grant in 2008 and became a Member of Accademia Nazionale dei Lincei in 2010. His research focuses on the synthesis of new functional materials, for applications in medicine and energy fields, in particular in spinal cord repair, splitting of water, and reduction of carbon dioxide into useful chemicals.

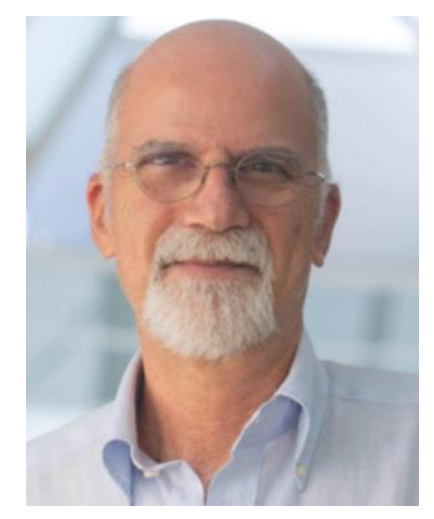

\section{Alberto Bianco}

Alberto Bianco received his PhD in 1995 from the University of Padova. As a scientist, he worked at the University of Lausanne, the University of Tu "bingen (Alexander von Humboldt fellow) and the University of Padova. He is currently Research Director at the CNRS in Strasbourg. His research interests focus on the design of multifunctional carbon-based nanomaterials for therapeutic, diagnostic and imaging applications. In 2017 he was elected as Fellow of the European Academy of Science, and in 2019 he received the CNRS Silver Medal. He is the co-author of over 250 papers. Since 2011 he has been the Editor of the journal CARBON.

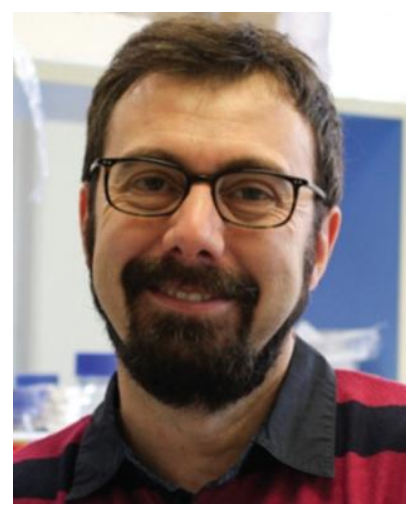

